\title{
A BAC-Based Physical Map of the Chicken Genome
}

\author{
Chengwei Ren, ${ }^{1,3}$ Mi-Kyung Lee, ${ }^{1,3}$ Bo Yan, ${ }^{1,3}$ Kejiao Ding, ${ }^{1}$ Bettye Cox, ${ }^{1}$ \\ Michael N. Romanov, ${ }^{2}$ Jennifer A. Price, ${ }^{2}$ Jerry B. Dodgson, ${ }^{2}$ and Hong-Bin Zhang ${ }^{1,4}$ \\ ${ }^{1}$ Department of Soil and Crop Sciences and Institute for Plant Genomics and Biotechnology, Texas A\&M University, \\ College Station, Texas 77843, USA; ${ }^{2}$ Department of Microbiology and Molecular Genetics, Michigan State University, \\ East Lansing, Michigan 48824, USA
}

\begin{abstract}
A genome-wide physical map constructed with bacterial artificial chromosomes (BACs) is an essential component in linking phenotypic traits to the responsible genetic variation in the genomes of plants and animals. We have constructed a physical map of the chicken genome from 57,091 BACs (7.9-fold haploid genome coverage) by restriction fingerprint analysis using high-resolution polyacrylamide gel electrophoresis. The physical map consists of 2331 overlapping BAC contigs and is estimated to span $1510 \mathrm{Mb}$ in physical length. BAC contigs were verified manually and by screening the BACs with 367 DNA markers. A total of 361 of the contigs have been anchored to the existing chicken genetic map. This map represents the first genome-wide, BAC-based physical map of the chicken genome. It provides a powerful platform for many areas of chicken genomics, including targeted marker development, fine mapping of genes and QTL alleles, positional cloning, analysis of avian genome organization and evolution, chicken-mammalian comparative genomics, and large-scale genome sequencing.

[Supplemental materials, including the three source BAC libraries, clone fingerprints, and contig map, are available online at http://hbz.tamu.edu. The database of BAC clones associated with DNA markers is available online at http: / / poultry.mph.msu.edu / resources/Resources.htm\# bacdata.]
\end{abstract}

The chicken (Gallus gallus) serves as both a major agricultural animal species and an important model organism for studies of developmental biology, genetics, and diseases of human and animals (Brown et al. 2003). The chicken also serves as a model for the molecular genetic analysis of all wild and domestic birds. Comparative mapping studies have revealed that the chicken genome demonstrates a surprising level of conserved gene order in comparison with mammalian genomes, especially that of the human (Burt et al. 1999; Groenen et al. 2000; Waddington et al. 2000; Suchyta et al. 2001). Preliminary sequence analysis has shown that the chicken genome provides a very informative comparison to mouse and human genomes to aid in the annotation of exons and conserved regulatory domains (Margulies and Green 2003). Thus, the chicken has recently been given high priority as a target for full-genome sequencing by the National Human Genome Research Institute (http://www.genome.gov/ page.cfm?pageID=10002154).

A critical component of complete genome-sequence assembly is the availability of a genome-wide, BAC-based physical map (Green 2001; Zhang and Wu 2001). It is desirable that BAC-based physical maps be integrated with other genome maps, in particular, genetic linkage maps, in which phenotypic trait alleles such as quantitative trait loci (QTL) can be placed. Integrated physical/ genetic maps are of importance for QTL fine mapping, highthroughput EST (expressed sequence tag) mapping, and effective positional cloning of genes, including those encoding QTL of economic importance (Zhang and Wing 1997; Zhang and $\mathrm{Wu}$ 2001). Progress in the development of local BAC contig maps for regions of the chicken genome has been reported (Crooijmans et al. 2003). However, no genome-wide, BAC-based physical map of the chicken genome has yet been described.

The chicken haploid genome is 1.14 Gigabase pairs $(\mathrm{Gb})$ in

${ }^{3}$ These authors contributed equally to this work.

${ }^{4}$ Corresponding author.

E-MAIL hbz7049@tamu.edu; FAX (979) 862-4790.

Article and publication are at http://www.genome.org/cgi/doi/10.1101/ gr.1499303. size (Bennett et al. 2003), organized as 38 pairs of autosomes and one pair of sex chromosomes (ZW). A consensus genetic linkage map has been generated for the chicken genome (Groenen et al. 2000; Schmid et al. 2000). This map contained 1965 loci ordered on 50 linkage groups and spanned $\sim 3800 \mathrm{cM}$. We report here a genome-wide, BAC-based physical map of the chicken genome. The map was developed from BAC clones selected randomly from three large-insert BAC libraries constructed from DNA of an inbred Red Jungle Fowl line (UCD001) female (ZW) bird (Lee et al. 2003) using the DNA-sequencing gel-based restriction fingerprinting method (Zhang and Wu 2001). Numerous physical map contigs have also been anchored to the existing chicken genetic map and the chicken-human comparative genetic map (Groenen et al. 2000).

\section{RESULTS AND DISCUSSION}

\section{BAC Fingerprinting}

We fingerprinted a total of 66,048 clones from the three complementary BAC libraries (Lee et al. 2003; Table 1) on 1032 autoradiographs using the DNA-sequencing gel-based restriction fingerprinting method (Zhang and Wing 1997; Tao and Zhang 1998; Chang et al. 2001; Tao et al. 2001; Zhang and Wu 2001). Of these fingerprints, 7969 clones (12.0\%) were deleted during fingerprint editing due to failures in standard DNA markers, insert-empty clones, or failed fingerprinting. In addition, 988 clones $(1.5 \%)$ were ignored by the FPC V6.0 program (http://www.genome. clemson.edu/fpc/faq.html; Soderlund et al. 2000) during contig assembly, because they contained four or fewer bands in the range of from 58 to $773 \mathrm{bp}$, providing insufficient information to be included in the contig assembly. Thus, a total of 57,091 clone fingerprints were used for contig assembly. These clones contain $\sim 7.9$-fold representation for the autosomes and 4.0-fold for each sex chromosome of the chicken genome. Studies (Zhang and Wing 1997; Chang et al. 2001; Tao et al. 2001; Xu et al. 2003) have demonstrated this redundancy to be sufficient for construction of a high-coverage physical map. The clones from the BamHI 
Table 1. The Source BACs Fingerprinted for the Chicken Physical Map

\begin{tabular}{lllccrc}
\hline Libraries & Cloning site & Vectors & $\begin{array}{c}\text { Mean insert } \\
\text { size (kb) }\end{array}$ & $\begin{array}{c}\text { No. of clones } \\
\text { fingerprinted }\end{array}$ & $\begin{array}{c}\text { Valid bands } \\
\text { per clone }^{\mathbf{b}}\end{array}$ & $\begin{array}{c}\text { Genome } \\
\text { coverage }\end{array}$ \\
\hline 031-JF256-BI & BamHI & pBeloBAC11 & 150 & 22,272 & 33.2 & $2.9 \times$ \\
032-JF256-RI & EcoRI & pECBAC1 & 152 & 20,736 & 35.2 & $2.8 \times$ \\
033-JF256-H3 & HindIII & pECBAC1 & 171 & 23,040 & 38.0 & $3.5 \times$ \\
& Total & & 158 & 66,048 & 35.6 & $9.2 \times$ \\
\hline
\end{tabular}

aFor details of the BAC libraries, see Lee et al. (2003) and http://hbz.tamu.edu.

${ }^{b}$ Valid bands are those in the range of 58 773 bp.

library, the EcoRI library, and the HindIII library had an average of $33.2,35.2$, and 38.0 bands per clone, respectively, in the range of from 58 to $773 \mathrm{bp}$ (Table 1).

\section{Contig Assembly and Manual Editing}

We used the computer program FPC V6.0 (Soderlund et al. 1997, 2000; http://www.genome.clemson.edu/fpc/faq.html) to assemble the physical map contigs of the chicken genome from BAC fingerprints. Previous research (Chang et al. 2001; The International Human Genome Mapping Consortium 2001; Tao et al. 2001) demonstrated that clones from different BAC libraries, despite differences in insert sizes, were assembled in an equivalent manner (also see Fig. 1), therefore, the clones of all three libraries were assembled together into the map contigs. Initially, a series of tests were performed to determine the parameters suited for contig assembly. On the basis of these results, a tolerance of 2 was selected for the assembly process.

We assembled the map contigs in three steps. First, a cutoff value of $1 \mathrm{e}-30$ was used for automatic contig assembly to limit the number of chimeric contigs arising from clones with many bands, which likely derived from an unusually high density of restriction sites for the fingerprinting enzyme. By this step, 12,446 of the clones $(22 \%)$ were assembled into 859 contigs, with an average of 45.7 bands per clone in the range of from 58 to 773 bp, comparing with 35.6 bands per clone of all 57,091 BACs. The higher stringency used at this step reduced the probability of mismatching BACs with high-density fingerprints into chimeric contigs.

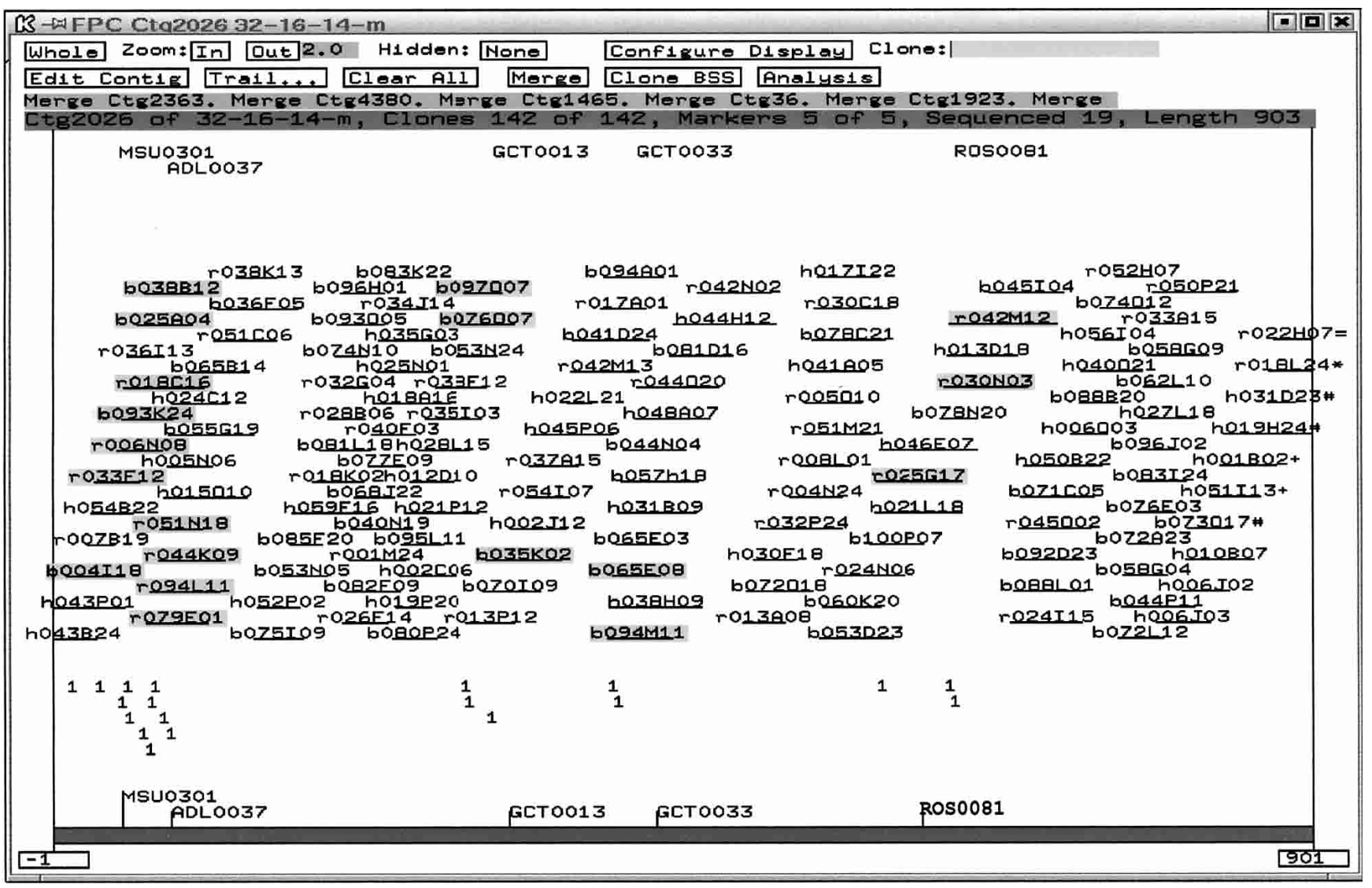

Figure 1 Example of a chicken BAC contig anchored to the chromosome 1 genetic map of the chicken genome. This contig consists of 142 clones from three source BAC libraries, contains 903 unique fingerprint bands, and is estimated to span $4.01 \mathrm{Mb}$. The clones prefixed with " $\mathrm{h}$ " were from the HindIII BAC library, with " $b$ " from the BamHI BAC library, and with " $r$ " from the EcoRI BAC library. The contig was anchored to the region around 361 CM of the chromosome 1 genetic map using five DNA markers, MSU0301, ADL0037, GCT0013, GCT0033, and ROS0081 (Groenen et al. 2000). The highlighted clones indicate the positive clones identified by DNA marker hybridization. 
Second, automatic contig assembly was performed at a cutoff of 1e-16. Chimeric contigs were split, and contigs with five or more questionable clones were rearranged at higher stringencies (lower cut-off values) using the Dqer function of the FPC V6.0. The Dqer automatically reanalyzed contigs with five or more questionable clones by reassembling them up to three times, where each time the cutoff was lowered by a factor of 10 . We ran the Dqer function a few times at cutoffs up to 1e-26 (by setting at 1e-23) to disassemble all contigs with five or more questionable clones. The singletons resulting from the Dqer function were automatically added at the stepwise cutoffs of $1 \mathrm{e}-26,1 \mathrm{e}-20$, and 1e-16. After this step, 3747 contigs were obtained and 7683 singletons remained.

Finally, at a cutoff of $1 \mathrm{e}-16$, each contig was analyzed manually using the FPC functions of the Calc CB map, the Fp Order CB map, the Contig window, and the Fingerprint window. Neighboring contigs were merged, false-overlapped clones (clones with too many extra bands) were removed, and the resulted singletons were again added manually. In situations in which the overlap between two contigs was significant, or in which DNA markers were available, we increased the cutoff values up to $1 \mathrm{e}-10$ for contig merger and singleton addition. As a result, 2331 contigs were obtained for the physical map of the chicken genome (Table 2). Figure 1 shows an example of a BAC contig anchored to the chromosome 1 genetic map of the chicken genome using five DNA markers (Groenen et al. 2000).

The physical length of the assembled 2331 contigs was $\sim 1510 \mathrm{Mb}$, based on 339,847 unique bands, with each being equivalent to $4.44 \mathrm{~kb}$ (Table 2). The total physical length of the contigs is larger than the estimated $1.14 \mathrm{~Gb}$ size of the chicken genome (Bennett et al. 2003) by 32\%, suggesting that most contigs actually overlap adjacent contigs, even though these overlaps were not detected with the present data and stringency used. This result is similar to the BAC contig physical mapping results of soybean (C. Wu, S. Sun, P. Nimmakayala, F.A. Santos, K. Meksem, R. Springman, K. Ding, D.A. Lightfoot, and H.-B. Zhang, in prep.; http://hbz.tamu.edu) and japonica rice (Y. Li, T. Uhm, C. Ren, C. Wu, T.S. Santos, M.-K. Lee, B. Yan, F.A. Santos, A. Zhang, Z. $\mathrm{Xu}$, et al., in prep.; http://hbz.tamu.edu) obtained in our labora-

\section{Table 2. Summary of the Chicken Physical Map}

\begin{tabular}{lr}
\hline Number of clones fingerprinted & 66,048 \\
Number of clones with successful fingerprints & 58,079 \\
Number of clones used for map contig assembly & 57,091 \\
Number of singletons & 6,830 \\
Number of contigs & 2,331 \\
>200 clones & 1 \\
$101-200$ clones & 29 \\
$51-100$ clones & 206 \\
$26-50$ clones & 476 \\
$10-25$ clones & 603 \\
$3-9$ clones & 812 \\
2 clones & 204 \\
Physical length of the contigs & $1,510 \mathrm{Mb}^{\mathrm{a}}$ \\
\hline
\end{tabular}

aEach fingerprint band was estimated to represent an average of 4.44 $\mathrm{kb}$. It was estimated by the average insert size of the BAC clones (158 $\mathrm{kb}$; Table 1; Lee et al. 2003) divided by the average number of valid bands per clone (35.6 bands per clone $=2,066,401$ bands in total $/$ 58,079 clones) used for the map contig assembly. The average insert size of the clones was estimated from the Notl digestion of 944 clones selected randomly from the three source BAC libraries, followed by pulsed-field gel electrophoresis (Lee et al. 2003). Unlike fingerprinting on agarose gels, the sample DNAs were double digested with HindIII and Haelll, but only end-labeled at the HindIII sites, so that the bands without HindIII sites were not shown on the autoradiographs by fingerprinting on polyacrylamide gels. tory. Therefore, it is expected that these contigs could be further merged and refined, as additional DNA marker and BAC endsequence data become available. The longest contig of the map contains 218 clones, encompassing 1240 unique bands, and spans $5.5 \mathrm{Mb}$ in physical length.

\section{Contig Reliability}

We evaluated the reliability of chicken BAC contigs using several approaches. First, we checked the consensus band maps of fingerprint order (clone order) for each contig (Fig. 2A). Of the 2331 contigs, only $201(8.6 \%)$ contained 1- 4 questionable clones (with $>50 \%$ of bands unmatched), most of which contained only one questionable clone. Contig score is another indicator of group alignment of all clones in a contig (Soderlund et al. 1997). Most of our contigs had a contig score ranging from 0.85 to 1.00 , whereas a small number of the contigs had a score between 0.80 and 0.85 . The overall band patterns in each contig were also checked for the number of extra bands. According to the FPC V6.0 program, 15 or fewer extra bands for each clone in a contig are acceptable for fingerprints generated by polyacrylamide gel electrophoresis (see FPC User's Guide for Version 6.0; http:// www.genome.clemson.edu/fpc/faq.html). If a clone in a contig was not a questionable clone, but had $>15$ extra bands (encountered only in a few cases in this study), the best match for that clone was determined, and the clone was removed, reorganized into another contig, or rearranged within the same contig as necessary. The second method used to evaluate contig reliability involved analysis of fingerprint patterns in the Fingerprint Window (Fig. 2B). We checked the fingerprint patterns of every contig to ensure that every clone within a contig was properly situated with respect to its most closely related neighbors, as defined by fingerprint similarity.

The third approach to contig verification involved screening the source BACs of the physical map using DNA markers (Table 3 ), primarily by the overgo hybridization method (Ross et al. 1999). If a contig was assembled properly, the BAC clones that hybridized to a single-copy overgo marker should be assembled to a segment of a single contig. Overgos designed from 367 DNA markers were used as probes to screen filter arrays of either the BamHI-derived or the EcoRI-derived source BAC libraries (Lee et al. 2003), obtaining a total of 1124 positive clones. Each marker detected 1-15 clones, with an average of 3.1 BACs per marker. Of the 1124 positive BACs, 1069 that were detected by 350 of the markers were integrated into 361 contigs. Of the 350 markers, $287(82 \%)$ had their positive clones located in single contigs, whereas 63 (18\%) markers hybridized to BACs located in two or more contigs. There are two major explanations for the assignment of BACs detected by a marker to two or more contigs. The first is false positive BAC identifications or repetitive overgo sequences. Because only very limited sequence data are available to date for the chicken genome, it is not always possible to eliminate repetitive overgo probes in advance. In particular, some gene or EST-based overgo sequences may detect multiple members of a gene family, although efforts were made in overgo design to minimize this possibility. The second explanation is that in several cases, two contigs detected by the same marker may, in fact, overlap in the region of the marker, but the overlap may be too short to be statistically significant in the fingerprint analysis. Several contigs of this sort were merged by increasing the cutoff values from $1 \mathrm{e}-16$ to $1 \mathrm{e}-10$, but we chose not to increase the cutoff value any further due to the potential risk of generating false positive mergers. Fifty-one contigs were found to contain two or more DNA markers (Table 3; Fig. 1). The locations of the common markers in the chicken consensus linkage map (Groenen et al. 2000; Schmid et al. 2000) were consistent with 
A

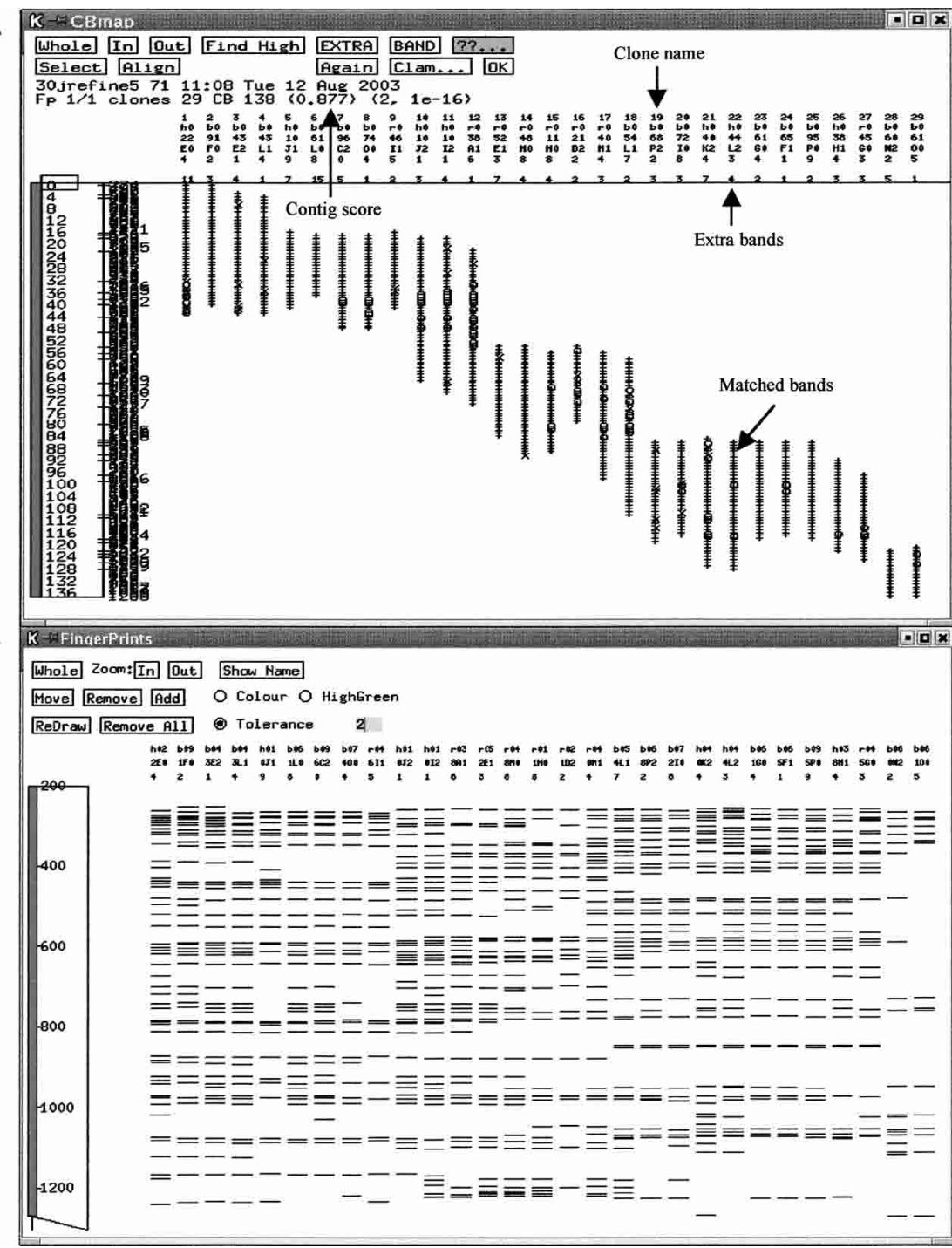

Figure 2 Example of a consensus band map $(A)$ and the clone order fingerprints $(B)$ of a BAC contig of the chicken physical map. These two maps are used to verify contig assemblies. The figure shows only part of the maps.

their assignments to a single BAC contig, suggesting that the relevant contigs were properly assembled in our map.

In conclusion, we constructed a genome-wide BAC contig map of the chicken genome, consisting of 2331 contigs that, together, span $1510 \mathrm{Mb}$. The BAC libraries that support this map (Lee et al. 2003) have been provided to numerous user groups, including the Washington University Genome Sequencing Center (http://genome.wustl.edu/), where BAC end sequences have been obtained from most of the clones. Therefore, this map will be integrated with, and provide a resource for assembly of the complete chicken genome sequence. The map also provides a platform for merging data collected by all of the BAC library user groups that will be critical to the annotation of the genome sequence and the application of that sequence to further research in molecular genetics and agriculture. Efforts continue to improve the map by hybridizing additional genetic markers, comparative genetic map markers, ESTs, and BAC end sequences to BAC filter arrays. All clones, clone fingerprints, and contigs of the physical map are available at our Web site: http://hbz.tamu.edu, and all contigs can be viewed as the Supplemental materials.

\section{METHODS}

\section{Source BAC Libraries}

Three BAC libraries were constructed using partial digests with three different restriction enzymes, as described previously (Lee et al. 2003; http://hbz.tamu.edu; Table 1). The DNA source for the BAC libraries was red blood cells from a single female bird (\#256) of the Red Jungle Fowl (Gallus gallus) inbred line UCD001.

\section{BAC Fingerprinting}

BAC DNA was isolated and fingerprinted according to Chang et al. (2001) and Tao et al. (2001). Briefly, BAC clones maintained in a 384-well microplate were inoculated in four 96-deep well plates containing $1 \mathrm{~mL} \mathrm{LB}$ medium per well plus $12.5 \mu \mathrm{g} / \mathrm{mL}(\mathrm{w} / \mathrm{v})$ chloramphenicol and grown at $37^{\circ} \mathrm{C}$ with shaking at $250 \mathrm{rpm}$ for $16-20 \mathrm{~h}$. BAC DNA was isolated and purified in the 96-deep well plates and then in 12-microtube strips using a modified alkaline lysis method (Chang et al. 2001; Tao et al. 2001). The DNA was double digested with HindIII and HaeIII and the HindIII fragments were end-labeled with $\left[{ }^{33} \mathrm{P}\right] \mathrm{dATP}$ using reverse transcriptase at $37^{\circ} \mathrm{C}$ for $2 \mathrm{~h}$, and then subjected to electrophoresis on $3.5 \%(\mathrm{w} / \mathrm{v})$ polyacrylamide DNA sequencing gels at $90 \mathrm{~W}$ for $100 \mathrm{~min}$, using the Sau3AI-digested, $\left[{ }^{33} \mathrm{P}\right] \mathrm{dATP}$ labeled $\lambda$ DNA as the molecular-weight standard. The gels were dried and autoradiographed.

\section{BAC Contig Assembly}

Fingerprint editing and contig assembly were conducted on a Dell Precision 330, with a $1.4 \mathrm{GHz} \mathrm{CPU}$, equipped with a Linux 7.0 platform. The fingerprint autoradiographs were scanned into image files using a UMAX Mirage D-16L scanner, and were digitized and edited using Image $3.10 \mathrm{~b}$ software (Sulston et al. 1989). Because of the lower resolution of the higher molecularweight bands at the top of the gels, only the bands ranging from 58 to $773 \mathrm{bp}$ were used for contig assembly. Vector bands were removed manually from the data files. Clones that failed in fingerprinting or that had no inserts were deleted manually. All digitized band data were standardized according to the known band sizes of the $\lambda$ DNA/Sau3AI markers and converted from base pairs into migration rates.

Contig assembly was done using the computer program FPC V6.0 (Soderlund et al. 2000; http://www.genome.clemson.edu/ fpc/faq.html). Band sizes in the range of from 773 to 58 bases were converted into migration rates of 258-1274. A series of tests were conducted in which the fingerprints of a set of overlapping clones were compared using different tolerances (from 1 to 7 ) and cutoffs $(1 \mathrm{e}-8 \sim 1 \mathrm{e}-30)$. On the basis of these tests, a fixed tolerance of 2 and a primary cutoff of $1 \mathrm{e}-16$ were selected for contig assembly.

\section{DNA Markers and BAC Library Screening}

BAC libraries, filter arrays, and procedures for hybridization with longer PCR-generated DNA fragments have been described (Lee et al. 2003). Overgo design, labeling, and hybridization were generally conducted as described by Ross et al. (1999). Sequencetagged sites used for overgo design were chosen from mapped markers and genes (Groenen et al. 2000; Schmid et al. 2000) and 
Table 3. Summary of DNA Markers Used for Contig Verification and Integration of the Physical Map Contigs with the Chicken Genetic Map

\begin{tabular}{lc}
\hline Number of positive clones in total: & 1124 \\
Positive clones in contigs & 1069 \\
Positive clones in singletons & 55 \\
Positive clones for each marker & $1-15$ \\
Positive clones per marker & 3.1 \\
Number of markers used in total: & 367 \\
Number of markers in contigs: & 350 \\
One marker in one contig & 287 \\
One marker in multiple contigs & 63 \\
Number of markers in singletons & 17 \\
Number of contigs containing DNA markers: & 361 \\
Contigs containing one marker & 310 \\
Contigs containing more than one marker & 51
\end{tabular}

${ }^{\mathrm{a} O n l y} \sim 1 / 3$ of the source BAC clones were screened with each DNA marker.

are listed at http://poultry.mph.msu.edu/resources/Resources. $\mathrm{htm} \#$ bacdata. Individual sequences used for overgos are available upon request. Overgo probe hybridizations were carried out in pools of 36 overgos at a time. A $6 \times 6 \times 6$ matrix of 216 overgo probes was hybridized in each of three dimensions (18 hybridizations with 36 overgos each). Six additional hybridizations with 36 of the overgos each were done as a redundant fourth dimension to minimize false negatives and resolve ambiguous positives. Details of the overgo screening process have been described (Romanov et al. 2003).

\section{ACKNOWLEDGMENTS}

This study was supported by the USDA/CSREES-National Research Initiative (Project numbers 99-35205-8566 and 200152100-11225) and by Regional Research funds from the USDACSREES National Animal Genome Research Program, NRSP-8.

The publication costs of this article were defrayed in part by payment of page charges. This article must therefore be hereby marked "advertisement" in accordance with 18 USC section 1734 solely to indicate this fact.

\section{REFERENCES}

Bennett, M.D., Leitch, I.J., Price, H.J., and Johnston, J.S. 2003. Comparisons with Caenorhabditis ( 100 Mb) and Drosophila ( 175 $\mathrm{Mb}$ ) using flow cytometry show genome size in Arabidopsis to be $\sim 157 \mathrm{Mb}$ and thus $\sim 25 \%$ larger than the Arabidopsis genome initiative estimate of $\sim 125 \mathrm{Mb}$. Ann. Botany 91: 547-557.

Brown, W.R.A., Hubbard, S.J., Tickle, C., and Wilson, S.A. 2003. The chicken as a model for large-scale analysis of vertebrate gene function. Nat. Rev. Genet. 4: 87-98.

Burt, D.W., Bruley, C., Dunn, I.C., Jones, C.T., Ramage, A., Law, A.S., Morrice, D.R., Paton, I.R., Smith, J., Windsor, D., et al. 1999. The dynamics of chromosome evolution in birds and mammals. Nature 402: 411-413.

Chang, Y.L., Tao, Q., Scheuring, C., Meksem, K., and Zhang, H.-B. 2001. An integrated map of Arabidopsis thaliana for functional analysis of its genome sequence. Genetics 159: 1231-1242.

Crooijmans, R., Dijkhof, R., Aerts, J., Hemmatien, K., Cornelissen, S., Veenendaal, T., van der Poel, J., and Groenen, M. 2003. A sequence ready BAC map of chicken chromosome 10. In Proc. Plant Anim. Genomes XI Conference, P225 (abstract). San Diego, CA..

Green, E.D. 2001. Strategies for the systematic sequencing of complex genomes. Nat. Rev. Genet. 2: 573-583.

Groenen, M.A.M., Cheng, H.H., Bumstead, N., Benkel, B.F., Briles, W.E., Burke, T., Burt, D.W., Crittenden, L.B., Dodgson, J.B., Hillel, J., et al. 2000. A consensus linkage map of the chicken genome. Genome Res.
10: $137-147$.

The International Human Genome Mapping Consortium. 2001. A physical map of the human genome. Nature 409: 934-941.

Lee, M.-K., Ren, C.W., Yan, B., Cox, B., Zhang, H.-B., Romanov, M., Sizemore, F.G., Suchyta, S.P., Peters, E., and Dodgson, J.B. 2003. Construction and characterization of three complementary BAC libraries for analysis of the chicken genome. Animal Genet. 34: $151-152$.

Margulies, E.H. and Green, E.D. 2003. Extracting biological information from multi-species genomic sequence comparisons. In Proceedings of the advances in genome biology and technology 2003 meeting, p.72 (abstract). Marco Island, FL.

Ross, M.T., LaBrie S., McPherson J., and Stanton V.P. 1999. Screening large-insert libraries by hybridization. In Current protocols in human genetics (eds. N.C. Dracopoli, J.L. Haines, B.R. Korf, D.T. Moir, C.C. Morton, C.E. Seidman, J.G. Seidman, and D.R. Smith), pp. 5.6.1-5.6.52. John Wiley and Sons, New York.

Romanov, M.N., Price, J.A., and Dodgson, J.B. 2003. Integration of animal linkage and BAC contig maps using overgo hybridization. Cytogenet. Genome Res. (in press).

Schmid, M., Nanda, I., Guttenbach, M., Steinlein, C., Hoehn, M., Schartl, M., Haaf, T., Weigend, S., Fries, R., Buerstedde, J.-M., et al. 2000. First report on chicken genes and chromosomes. Cytogenet. Cell Genet. 90: 169-218.

Soderlund, C., Longden, I., and Mott, R. 1997. FPC: A system for building contigs from restriction fingerprinted clones. CABIOS 13: $523-535$.

Soderlund, C., Humphray, S., Dunham, A., and French, L. 2000. Contigs built with fingerprints, markers, and FPC V4.7. Genome Res. 10: $1772-1787$.

Suchyta, S.P., Cheng, H.H., Burnside, J., and Dodgson, J.B. 2001. Comparative mapping of chicken anchor loci orthologous to genes on human chromosomes 1, 4 and 9. Animal Genet. 32: 12-18.

Sulston, J., Mallett, F., Durbin, R., and Horsnell, T. 1989. Image analysis of restriction enzyme fingerprint autoradiograms. Bioinformatics 5: 101-106.

Tao, Q. and Zhang, H.-B. 1998. Cloning and stable maintenance of DNA fragments over $300 \mathrm{~kb}$ in Escherichia coli with conventional plasmid-based vectors. Nucleic Acids Res. 26: 4901-4909.

Tao, Q., Chang, Y.L., Wang, J., Chen, H., Islam-Faridi, M.N., Scheuring, C., Wang, B., Stelly, D.M., and Zhang, H.-B. 2001. BAC-based physical map of the rice genome constructed by restriction fingerprint analysis. Genetics 158: 1711-1724.

Waddington, D., Springbett, A.J., and Burt, D.W. 2000. A chromosome based model to estimate the number of conserved segments between pairs of species from comparative genetic maps. Genetics 154: $323-332$.

Xu, Z., Sun, S., Covaleda, L., Ding, K., Zhang, A., and Zhang, H.-B. 2003 Whole-genome physical mapping with BACs: Fingerprinting methods, source clone genome coverage, and map contig accuracy and size. In Proc. Plant Animal Genomes XI Conference, W224 (abstract). San Diego, CA.

Zhang, H.-B. and Wing, R.A. 1997. Physical mapping of the rice genome with BACs. Plant Mol. Biol. 35: 115-127.

Zhang, H.-B. and Wu, C. 2001. BAC as tools for genome sequencing. Plant Physiol. Biochem. 39: 195-209.

\section{WEB SITE REFERENCES}

http://hbz.tamu.edu; GENEfinder Genomic Resources, Texas A\&M University.

http://genome.wustl.edu/; Genome Sequencing Center, Washington University in St. Louis.

http://poultry.mph.msu.edu/; US Poultry Genome Project, Michigan State University.

http://www.genome.clemson.edu/fpc/faq.html; Genomics Institute, Clemson University.

http://www.genome.gov/page.cfm?pageID=10002154; Status of organisms in the prioritization process for genome sequencing and their 'White Paper' proposals, National Human Genome Research Institute, NIH.

Received May 5, 2003; accepted in revised form September 3, 2003. 


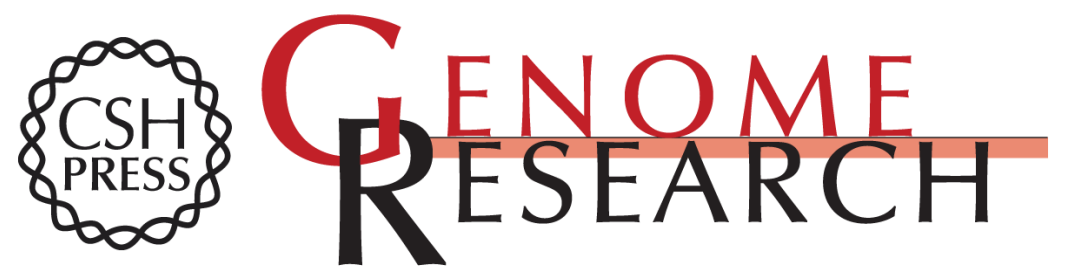

\section{A BAC-Based Physical Map of the Chicken Genome}

Chengwei Ren, Mi-Kyung Lee, Bo Yan, et al.

Genome Res. 2003 13: 2754-2758

Access the most recent version at doi:10.1101/gr.1499303

References This article cites 16 articles, 5 of which can be accessed free at:

http://genome.cshlp.org/content/13/12/2754.full.html\#ref-list-1

License

Email Alerting Receive free email alerts when new articles cite this article - sign up in the box at the Service top right corner of the article or click here.

\section{Affordable, Accurate} Sequencing.

To subscribe to Genome Research go to: https://genome.cshlp.org/subscriptions 